\title{
Performance Analysis of Primary User Aware Heuristic Dynamic Spectrum Channel Allocation
}

\author{
Deepshikha Bhati \\ Banasthali Vidyapeeth, Jaipur, India
}

\author{
Poonam Kshatriya \\ Banasthali Vidyapeeth, Jaipur, India
}

\begin{abstract}
In cognitive radio networks, the role of the medium access management layer is incredibly necessary since it permits secondary users to access the spectrum while not moving Primary Users' communications. Secondary users' and first users' pure mathematics has a bearing on the performance of the spectrum sharing algorithms. Also, secondary users' quality changes the topology of the network similarly as interference between the primary and secondary users. The situation of multiuser multichannel psychological feature radio networks introduces new challenges like co-channel interference. Consequently, the ability budget ought to be allotted to the secondary users subject to specific constraints. Hence, completely different secondary users can have different power and interference limits betting on the activity of primary users and on that secondary users are inflicting cochannel interference to every different. additionally, sanctioning Energy gathering in psychological feature radio networks is promising to increase their time period in order that the hybrid interweave/underlay access theme is adopted, which implies that secondary users will access the active and non-active primary user bands.
\end{abstract}

In this analysis paper, a best primary user aware heuristic dynamic spectrum allocation technique is projected. The study of impact of the subsequent factors: quality of the secondary users, spectrum quality, the primaryexclusive regions (PERs), the geographical locations of the nodes, property of secondary users, correlate shadow weakening, and also the activity of each primary users and secondary users.

\section{Keywords}

Cognitive radio networks

\section{INTRODUCTION}

Even though spectrum is changing into more and more scarce, spectrum occupancy rates area unit terribly low $[1,2]$. New technologies area unit presently being adopted to beat wireless spectrum shortages. Cognitive Radio has been projected so as to leverage spectrum utilization efficiencies and communication liableness through adaptation of operational parameters, continuous learning and possessing awareness of encompassing environments and activities [3].Within the cognitive radioframework, Secondary Users area unit allowed to opportunistically access the commissioned spectrum of the first Users, only if the interference level is below an appropriate threshold. If the interference condition isn't glad, genus secondary users should evacuate the channel like a shot. To make sure that such criteria area unit met, seamless schemes to dynamically access the spectrum area unit important for cognitive radio network.

Secondary users will access the spectrum victimization one among the subsequent schemes: distort, underlay, or overlay $[4,5]$, asdelineated in Fig. 1. In adistort theme, genus secondary users aren't allowed to cause any interference to the primary users and genus secondary users will access the vacant channels solely. Thus, the cognitive radio network should keep a watch on the activity of the plutonium and like a shot vacate the channel once a primary user becomes active and move to a different obtainable channel. The construct of moving between the obtainable White areas is said as spectrum quality [1].

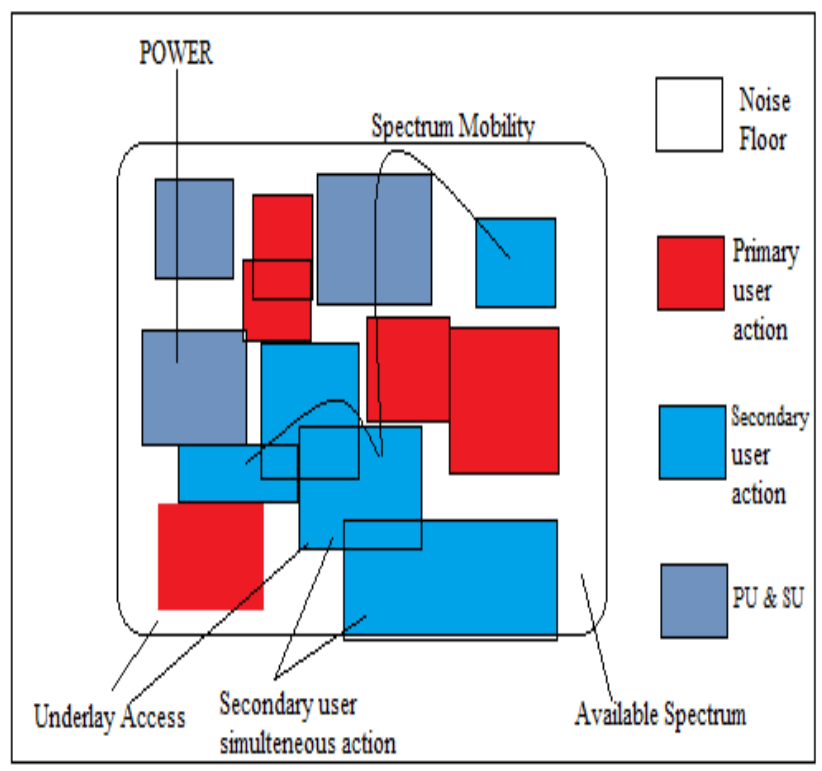

Fig. 1. Spectrum Access Schemes

In associate underlay theme, secondary users allowed to share the channel with a full of life chemical element given that secondary user interference levels don't exceed a suitable threshold. The utmost acceptable interference level is usually noted as interference temperature [6]. This may offer the cognitive radio network access to the spectrum at any time with the value of proscribing the transmission power in an exceedingly method that forestalls harmful interference to the primary users.

The overlay theme permits secondary user $\mathrm{s}$ to at the same time access the spectrum at the side of the primary users given that the cognitive radio network implements associate applicable committal to writing technique to mitigate the interference caused to the first network [7]. During this case, the cognitive radio network needs to have data concerning the code books or maybe messages happiness to the first network which can raise security issues. Every of the three access schemes have their advantages and prices.

\section{LITERATURE SURVEY}

Over the past few years, there has been important progress among the spectrum regulation domain to upset the growing demands of radio communication services. The first cognitive radio-based customary, IEEE 802:22, might be a centralized one where a CRBS is acting as a result of the management 
unit. Moreover, the CRBS might have data regarding the wireless atmosphere, users' activities, and type of transmitted data. Creating the foremost of such data among the resource allocation is anticipated to reinforce the state between the networks; thus, the algorithmic rule efficiency will increase. There are a unit pair of amendments of the standard: IEEE 802:22a and so the IEEE 802:22b amendments [8]. The IEEE 802:22a modification proposes standardization for management and management interfaces and IEEE 802:22b modification discusses supporting broadband services and observance applications, like supporting an outsized vary of low energy units and completely totally different QoS classes.

Another example of wireless standards for psychological feature radio technology is IEEE 802:11.(af, ac, n) series of standards that square measure planned to support the conventional 802:11 Wireless native house Network (WLAN) services $[9,10]$. Also, IEEE 1900:x series of standards aim to stipulate a standardized framework for radio resource management in future wireless systems [11-13]. Another standardization effort is that of the EU Telecommunications Standards Institute (ETSI) to manage the accredited shared access among future Evolution (LTE) operators among the band 2:3 GHz per second and 2:4 GHz per second [14]. Moreover, IEEE 802.19 customary goes to line the framework for state of the many unauthorized systems like 802:11af, $802: 22$, and 802:15:4 [15].

Autonomous spectrum allocation algorithms square measure planned in [16-20], where the spectrum access is accomplished by achieving individual goals rather like the QoS requirements or the energy consumption of a given secondary user. In $[16,17]$, the most target is on computing the minimal secondary user's transmission power that satisfies the individual secondary users'QoS goals. The algorithmic rule planned in [18] employs Stackelberg's theory to calculate the optimum resource allocation, whereas the algorithmic rule planned in [19] selects the secondary user mix with the perfect Signal to Noise quantitative relation (SNR) to utilize the lowest transmission power. Paper [20] presents a pricingbased non-cooperative game model for power management by secondary users. The target is to supply turnout fairness among these users whereas guaranteeing a minimum Signal to Interference and Noise quantitative relation at the secondary receiver.

In [21], the authors propose a scalable waterproof protocol for heterogeneous machineto- machine networks. The planned protocol achieves ranked performance by mistreatment completely totally different competitive priorities and incorporates every the persistent Carrier Sense Multiple Access (CSMA) and Time Division Multiple Access (TDMA) schemes. Moreover, a progressive competition priority theme is used to make sure honest access among multiple heterogeneous devices.

A DAB waterproof protocol for psychological feature radio networks (CMAC) is planned in [22] where each secondary user is supplied with one transceiver to find primary users' activities in its vicinity and then SU shares its sensing data with various secondary users. CMAC divides the timeframe into a pair of parts: the beacon quantity and so the data transfer quantity. Each secondary user periodically visits a typical management channel to induce data regarding PUs' activities.

In [23], authors introduce a DAB opportunistic Spectrum MAC, among that secondary users that want to talk with each other square measure classified on to make a cluster. Cluster heads square measure in charge for effort the traffic load data of a channel and for propagating this data at intervals their many clusters.

OS-MAC uses a probabilistic channel selection theme to cut back the inter-cluster interference. However, interference caused by primary user $\mathrm{s}$, that would be a key role of psychological feature radio, has not been enforced in OSMAC.

\section{SPECTRUM ALLOCATION SCHEMES FOR COGNITIVE RADIO NETWORKS}

Cognitive radio networks have the flexibility to reconfigure and adapt their computer code elements and architectures, so enabling edible delivery of broad services, additionally as sustaining sturdy operation beneath extremely dynamic conditions [5]. The pure mathematics of the secondary users and Pus has an impression on the performance of the spectrum sharing algorithms. Since primary user s have the correct of claiming the spectrum whenever required, it's crucial to make sure that primary users' satisfaction and QoS don't seem to be wedged by SUs' activity on the shared bands.

\section{(A) Network Model}

The cognitive radio network is assumed to possess $\mathrm{M}$ secondary users and one CRBS that is assumed to remember of primary user s' activity. This can be created doable by using some sensing techniques [24]. The CRBS is found within the middle of the operational space and its main duty is to coordinate the assignment of the channels and optimize the spectrum allocation. Each the cognitive radio network and also the primary network area unit set in shut proximity and also the topology of every of the networks follows a homogenized Poisson purpose method with nodes' density of $\beta_{P U}$ and $\beta_{S U}$ for the first and secondary networks, severally [25]. The transmission power of secondary user $s$ and first user s area unit stated as PSU and PPU severally. The system is assumed to possess ¥ PUs wherever every primary user is assigned one channel with a information measure B. Hence, the quantity of channels is adequate to the quantity of primary user $\mathrm{s}$. The cognitive radio network physical layer is assumed to be Filter Bank Multi-Carrier. The interference from alternative primary user $\mathrm{s}$ on the channels that they're not transmittal on is taken into account negligible.

This can be even by the actual fact that Filter Bank MultiCarrier has very tiny aspect lobe that considerably reduces the interference [26]. Also, Channel State data and sensing results area unit assumed to be sent from secondary user $\mathrm{s}$ to the CRBS employing a dedicated management Channel that's not tormented by the activity of primary users.

\section{(B) Traffic Flow Model}

In order to increase the efficiency of the dynamic spectrum allocation algorithm and reduce the collision ratebetween SUs, the operating time is divided into time slots where secondary users are allowed tobegin transmitting only at the start of any time slot. The communications traffic flowsof the primary users and secondary users are modelled as Bernoulli arrival processes with parameters $\lambda_{P U}$ and $\lambda_{S U}$, respectively [27]. Bernoulli process is the discrete-time analog of the Poissonarrival process where the arrivals of the packets can take place at some time slot k.Mathematically, the traffic of a secondary user can be described as a point process consisting ofa sequence of arrival instants $\{\mathrm{t} 1 ; \mathrm{t} 2 ; \mathrm{tm}\}$ measured from the 
origint $t_{0}=0$.The number of arrivals for slot $\mathrm{k}$ follows a binomial distribution:

$P\left\{N_{k}=n\right\}=\left(\frac{k}{n}\right) \lambda_{S U}^{n}\left(1-\lambda_{S U}\right)^{(k-n)} \quad$ eq. (1)

Also, the number of time slots between two arrivals is geometrically distributed withparameter $\lambda_{S U}$ and the probability of having $\mathrm{O}$ packets arriving at the same timeslotis given by:

$P\left\{A_{n}=O\right\}=\lambda_{S U}\left(1-\lambda_{S U}\right)^{O}, O \in N \quad$ eq.

Under this model, communication sessions are allowed to begin at the start of anytime slot and if a secondary usergenerates a packet before the start of a time slot, this packet has to be stored in a local buffer and the secondary userwould wait till the next time slot to contendfor a channel. The secondary user then will initiate a communication session if it is grantedaccess. It is important to note that the change in the primary users' communication behavior does not happen frequently and can be assumed to be fixed for a minimum durationof one time slot. Hence, the sensing information is valid for at least one time slot.

Fig. 1 shows the activity of a node that has traffic intensity of 0.5 , which meansthat this node will be producing packets with a probability of 0.5 at each time-slot.This node is producing one packet at time slots $1,3,5$ and 8 , and two packets at timeslot 2 .

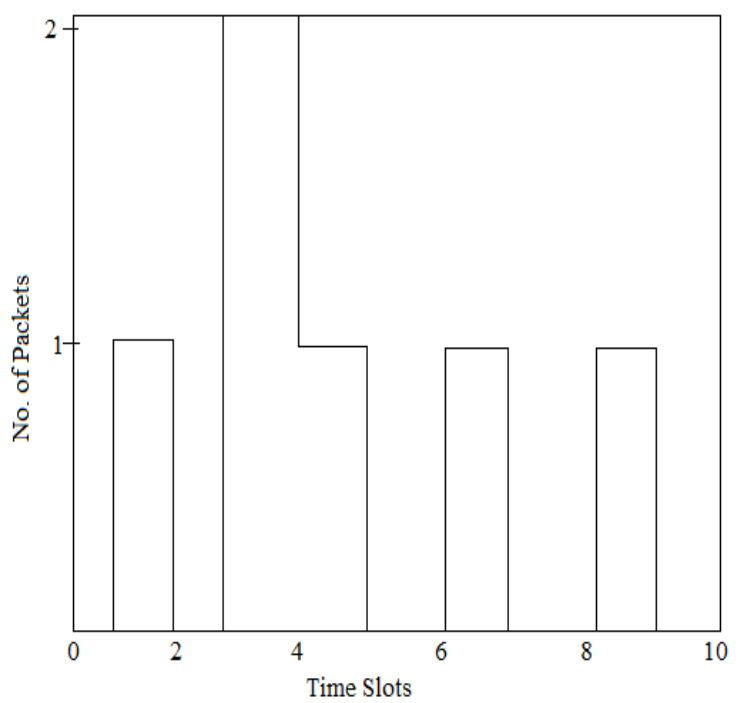

Fig.1. Packet arrival from a node that has traffic intensity of 0.5

\section{PROPOSED PU-AWARE HEURISTIC DYNAMIC SPECTRUM ALLOCATION ALGORITHM}

The problem developed within the previous section may be a binary number non-linear programming that is tough to unravel. As an alternative, the binary optimization variable is relaxed so the new downside is solved exploitation the primaldual interior-point methodology. Such AN approach still needs Brobdingnag Ian process resources to search out the answer for 6 sets of Lagrangian multipliers, however.

To overcome this obstacle, this paper proposes a PU-Aware Heuristic Dynamic Spectrum Allocation algorithmic program that gives a satisfactory protection level to the first users whereas quickly assignment the obtainable bands to the secondary users. The algorithmic program can use each distort and underlay access schemes.First, let us define the expected interference caused by $S U_{i}$ to $P U_{j}$ given the mutualEuclidean distance between them as follows:

$E_{i j}\left\{I_{0} \mid S(i, j)\right\}=G_{0}^{j}(i, j)^{-\eta} \exp \left(\frac{1}{2}\left(\sigma_{i j} \frac{\log 10}{10}\right)^{2}\right) P_{S U}^{i}$

Next, let $\exists$ be the cost function where $\exists_{i j}$ is the cost of assigning channel $\mathrm{j}$ to $S U_{i} . \exists_{i j}$ is defined as follows:

$\exists_{i j}=$
$\left(\left(L N \mid S(i, j)<R_{0}+\epsilon\right)(\sqrt{2}-\varphi(i, j)) E_{i j}\left\{I_{0} \mid S(i, j)\right\}+0.6 P A^{j}+\left(S A^{i}+1\right)^{-1}\right) \forall i, j$ eq. (4)

where $\mathrm{LN}$ is a large number that is greater than unit value, $\varphi(i, j)$ is the normalized euclidiandistance between secondary user $S U_{i}$ and primary user $P U_{j}$, and $S A^{i}$ is the activity of $S U_{i}$. The term $\left.L N \mid S(i, j)<R_{0}+\epsilon\right)$ increases the cost of assigning channel $\mathrm{j}$ to $S U_{i}$ considerably,if it is close to $P U_{j}$. Also, the term $\sqrt{2}-\varphi(i, j)$ ensures maximizing the Euclidiandistance between secondary users and primary users that will be transmitting on the same channel. Forexample, when $\varphi(i, j)$ has a high value, this means that $S U_{i}$ and $P U_{j}$ are far awayfrom each other, and hence the cost will be reduced. On the other hand, as $\varphi(i, j)$ decreases, the cost will increase. The second term in eq. (4), $0.6 P A^{j}$, ensures use of an interweave scheme first by adding an extra cost for invoking the underlay scheme.

The last term represents the activity of secondary users; if $S U_{i}$ is very active, it will get a higherpriority to access the spectrum.

\section{RESULTS \& DISCUSSION}

The performance analysis of the planned dynamic spectrum allocation rule is evaluated victimization the chance of success parameter that is that the chance that secondary users with information to transmit reach obtaining channels assigned to them. The correlation of the shadow weakening map is about to $l=20$ and also the path loss exponent $(\boldsymbol{\eta})$ is about to four. Also, the communication activity of the first users $\left(\lambda_{P U}\right)$ is about to 0.5 . The performance of the planned rule is compared to the rule: a carrier sense multiple access with collision rejection (CSMA/CA) based mostly algorithm.

Fig. two shows the result of secondary users' communication traffic $\left(\lambda_{S U}\right)$ on once the quantity of accessible channels is thirty. The PU-Aware Heuristic Dynamic Spectrum Allocation rule (PUAH-DSA) perpetually outperforms the CSMA/CA-based rule for all values of $\lambda_{S U}$. Once PER is taken into thought, the performance can slightly degrade, particularly once $\lambda_{S U}>0.3$ for $\beta_{S U}=50$ and once $\lambda_{S U}>0.7$ for $\beta_{S U}=30$. This is often as a result of the inflated demand from secondary users that reciprocally results in assignment channels to some secondary users even though they're set close to the active PUs. Moreover, the PU-Aware Heuristic Dynamic Spectrum Allocation rule outperforms the interweave-only rule once $\lambda_{S U}$ is a smaller amount than 0.5 . Once $\lambda_{S U}$ is larger than 0.5 , the interweave-only rule are a much better possibility. This is often as a result of the very fact that the PU-aware heuristic dynamic spectrum allocation rule puts the protection of primary users' communications initial no matter however this could have an effect on secondary users'QoS as a result of rejecting their access to the spectrum

As expected, once the quantity of channels will increase, the performance of all of the two algorithms improves. Also, the 
PU-aware heuristic dynamic spectrum allocation rule outperforms each of the opposite algorithms and might reach a hit rate of ninety fifth in cases wherever the quantity.

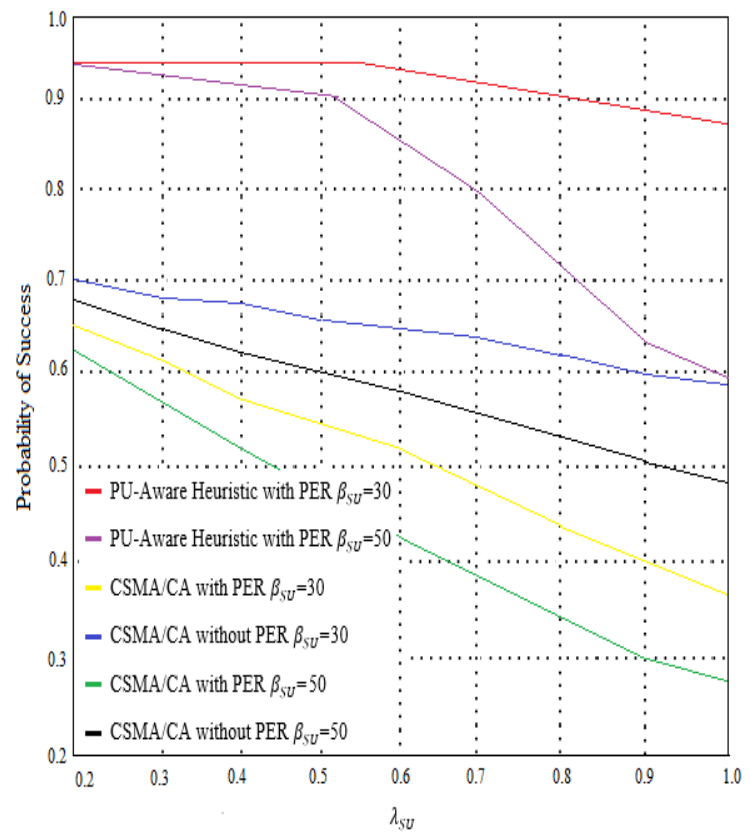

Fig. 2 Success probability vs. $\lambda_{s U}$ for different $\lambda_{s U}$ values with and without Primary Exclusive Region

\section{CONCLUSION \& FUTURE SCOPE}

From fig. 2, it can be conclude that proposed primary user aware heuristic dynamic spectrum allocation technique performed better as compared to carrier sense multiple access with collision avoidance technique.The results demonstrate a high success level in fulfilling psychological feature radio networks' demands for spectrum access whereas protective the QoS of the first network. Simulation results have conjointly shown that the projected algorithmic program outperforms ancient spectrum allocation algorithms. Once the amount of secondary users is far larger than the amount of obtainable channels, the performance of the projected algorithmic program degrades. this can be attributable to the actual fact that chemical element aware heuristic dynamic spectrum allocation algorithmic program puts the protection of primary users' communications because the initial and foremost priority that comes even before satisfying the wants of secondary users to access the spectrum. A recommended approach to be utilized in such a situation is grouping secondary users into totally different sets wherever every set will have solely a little of the active secondary users. In future double dynamic thresholding thought are often applied to boost channel allocation and chance of success.

\section{REFERENCES}

[1] M. Ibnkahla, Cooperative Cognitive Radio Networks: The Complete SpectrumCycle. CRC Press, 2014.

[2] A. Martian, I. Marcu, and I. Marghescu, "Spectrum occupancy in an urbanenvironment: A cognitive radio approach," in 6th Advanced International Conference on Telecommunications (AICT), 2010, pp. 25-29.

[3] M. Ibnkahla, Wireless sensor networks: A cognitive perspective. Boca Raton:CRC Press, Taylor \& Francis Group, 2013.
[4] A. Goldsmith, S. Jafar, I. Maric, and S. Srinivasa, "Breaking spectrum gridlockwith cognitive radios: An information theoretic perspective," Proceedings of theIEEE, vol. 97, no. 5, pp. 894-914, 2009.

[5] A. El-Mougy, M. Ibnkahla, G. Hattab, and W. Ejaz, "Reconfigurable wirelessnetworks,"Proceedings of the IEEE, vol. 103, no. 7, pp. 1125-1158, 2015.

[6] J. Mitola and G. Q. Maguire, "Cognitive radio: Making software radios morepersonal," IEEE Personal Communications, vol. 6, no. 4, pp. 13-18, 1999.

[7] Q. Li, S. H. Ting, A. Pandharipande, and Y. Han, "Cognitive spectrum sharingwith two-way relaying systems," IEEE Transactions on Vehicular Technology,vol. 60, no. 3, pp. 1233-1240, 2011.

[8] C.-W. Pyo, X. Zhang, C. Song, M.-T. Zhou, and H. Harada, "A new standardactivity in IEEE 802.22 wireless regional area networks: Enhancementfor broadband services and monitoring applications in TV whitespace," in $15^{\text {th }}$ International Symposium on Wireless Personal Multimedia Communications(WPMC), 2012, pp. 108-112.

[9] A. B. Flores, R. E. Guerra, E. W. Knightly, P. Ecclesine, and S. Pandey, "IEEE802.11 af: A standard for TV white space spectrum sharing."IEEE Communications Magazine, vol. 51, no. 10, pp. 92-100, 2013.

[10] IEEE Draft Standard for Information Technology Telecommunications and Information Exchange between Systems - Local and Metropolitan Area Networks Specific Requirements - Part 11: Wireless LAN Medium Access Control (MAC)and Physical Layer (PHY) Specifications Amendment 5: TV White Spaces Operation, Std., 2013.

[11] H. Harada, "White space communication systems: An overview of regulation,standardization and trial," Institute of Electronics, Information and Communication Engineers (IEICE) Transactions on Communications, vol. 97, no. 2 pp. 261-274, 2014.

[12] 1900.1a IEEE Standard Definitions and Concepts for Dynamic Spectrum Access: Terminology Relating to Emerging Wireless Networks, System Functionality, and Spectrum Management, Std., 2012.

[13] IEEE Standard for a Convergent Digital Home Network for Heterogeneous Technologies Amendment 1: Support of New MAC/PHYs and Enhancements, Std. 2015.

[14] J. Khun-Jush, P. Bender, B. Deschamps, and M. Gundlach, "Licensed sharedaccess as complementary approach to meet spectrum demands: Benefits fornext generation cellular systems," in ETSI Workshop on reconfigurable radiosystems, 2012, pp. 1-7.

[15] T. Baykas, J. Wang, M. Rahman, H. Tran, C. Song, S. Filin, Y. Alemseged,C. Sun, G. Villardi, C.-s. Sum et al., "Overview of TV white spaces: Currentregulations, standards and coexistence between secondary users," in IEEE $21^{\text {st }}$ International Symposium on Personal, Indoor and Mobile Radio Communications Workshops (PIMRC), 2010, pp. 38-43.

[16] Y. Huang, Q. Li, W.-K. Ma, and S. Zhang, IRobust multicast beamforming forspectrum sharing-based cognitive radios," IEEE Transactions on Signal Processing, vol. 60, no. 1, pp. 527-533, 2012. 
[17] J. Tadrous, A. Sultan, and M. Nafie, "Admission and power control for spectrumsharing cognitive radio networks," IEEE Transactions on Wireless Communications, vol. 10, no. 6, pp. 1945-1955, 2011.

[18] R. Xie, F. R. Yu, and H. Ji, "Energy-efficient spectrum sharing and power allocationin cognitive radio femtocell networks," in IEEE International Conferenceon Computer and Communication (INFOCOM), 2012, pp. $1665-1673$

[19] T. W. Ban, W. Choi, and D. K. Sung, "Capacity and energy efficiency of multiuserspectrum sharing systems with opportunistic scheduling,"IEEE Transactions on Wireless Communications, vol. 8, no. 6, pp. 2836-2841, 2009.

[20] X. Xie, H. Yang, A. V. Vasilakos, and L. He, "Fair power control using gametheory with pricing scheme in cognitive radio networks, "Journal of Communications and Networks, vol. 16, no. 2, pp. 183-192, 2014.

[21] Y. Liu, C. Yuen, X. Cao, N. U. Hassan, and J. Chen, "Design of a scalablehybrid MAC protocol for heterogeneous M2M networks," IEEE Internet ofThings Journal, vol. 1, no. 1, pp. 99-111, 2014.

[22] C. Cordeiro and K. Challapali, "C-MAC: A cognitive MAC protocol for multichannelwireless networks," in 2nd IEEE International Symposium on NewFrontiers in Dynamic Spectrum Access Networks, 2007, pp. $147\{157$.

[23] B. Hamdaoui and K. Shin, OS-MAC: "An efficient mac protocol for spectrumagilewireless networks," IEEE Transactions on Mobile Computing, vol. 7, no. 8,pp. 915-930, 2008.

[24] G. Hattab and M. Ibnkahla, "Multiband spectrum access: Great promises forfuture cognitive radio networks," Proceedings of the IEEE, vol. 102, no. 3, pp. 282-306, 2014

[25] A. Sabbah and M. Ibnkahla, "Efficient spectrum allocation algorithm for cognitiveradio networks in a shadow fading environment," in IEEE Wireless Communications and Networking Conference Workshops (WCNCW), 2014, pp. 288-293.

[26] B. Farhang-Boroujeny and R. Kempter, "Multicarrier communication techniquesfor spectrum sensing and communication in cognitive radios," IEEECommunications Magazine, vol. 46, no. 4, pp. 8085, 2008.

[27] V. S. Frost and B. Melamed, "Traffic modeling for telecommunications networks,"IEEE Communications Magazine, vol. 32, no. 3, pp. 70-81, 1994. 